\title{
Abnormal Growth Hormone
}

National Cancer Institute

\section{Source}

National Cancer Institute. Abnormal Growth Hormone. NCI Thesaurus. Code C78327.

A laboratory test result which indicates abnormal levels of growth hormone in a biologic specimen. 